\title{
Relationships Between Thyroid Hormones, Insulin-Like Growth Factor-1 and Antioxidant Levels in Hypothalamic Amenorrhea and Impact on Bone Metabolism
}

Authors

Antonio Mancini ${ }^{1,2}$, Edoardo Vergani ${ }^{1,2}$, Carmine Bruno ${ }^{1,2}$, Angelina Barini ${ }^{3,4}$, Andrea Silvestrini ${ }^{3,4}$, Elisabetta Meucci ${ }^{3,4}$, Calogero Messana5,6, Daniela Romualdi5,6, Rosanna Apa ${ }^{5,6}$, Antonio Lanzone ${ }^{5,6}$

Affiliations

1 UOC Endocrinologia e Diabetologia, Fondazione Policlinico Universitario A, Gemelli IRCCS, Rome, Italy

2 Istituto di Patologia Speciale Medica e Semeiotica Medica, Università Cattolica del Sacro Cuore, Rome, Italy

3 Fondazione Policlinico Universitario A, Gemelli IRCCS, Rome, Italy

4 Istituto di Biochimica e Biochimica Clinica, Università Cattolica del Sacro Cuore, Rome, Italy

5 UOC Ostetricia e Patologia Ostetrica, Fondazione Policlinico Universitario A, Gemelli IRCCS, Rome, Italy

6 Istituto di Clinica Ostetrico e Ginecologica, Università Cattolica del Sacro Cuore, Rome, Italy

Key words

amenorrhea, osteoporosis, non-thyroidal illness, osteocalcin, antioxidants

received $\quad 15.11 .2018$

accepted $\quad 11.02 .2019$

Bibliography

DOI https://doi.org/10.1055/a-0859-4285

Published online: 7.3.2019

Horm Metab Res 2019; 51: 302-308

(c) Georg Thieme Verlag KG Stuttgart · New York

ISSN 0018-5043

Correspondence

Prof. Antonio Mancini

Division of Endocrinology,

Catholic University of the Sacred Heart,

Largo A. Gemelli 8, 00168 Rome, Italy

Tel.: + 39/06/3015 4440, Fax: + 39/06/3015 7232

antonio.mancini@unicatt.it
丹 Supplementary Material for this article is available online at http://www.thieme-connect.de/products

\section{ABSTRACT}

Reduced bone mineral density (BMD) in Functional Hypothalamic Amenorrhea (FHA) is mainly related to hypoestrogenism, but other hormonal derangement (reduced conversion of T4-T3 and $\mathrm{GH}$ resistance) can play a role. These hormones are involved in antioxidant systems regulation. We evaluated the impact of hormonal alterations, with special focus on low T3 and IGF-1 levels, on antioxidant systems as a link with osteoporosis in FHA. Forty-three FHA patients, 15-34 years, with BMI range 17.3$23.4 \mathrm{~kg} / \mathrm{m}^{2}$, were divided in 2 groups according to fT3 levels; group $A(n=22)$, low fT3 $(<2.4 \mathrm{pg} / \mathrm{ml})$ and group $B(n=21)$, normalfT3 $(\geq 2.4 \mathrm{pg} / \mathrm{ml})$. We evaluated hormonal parameters (fT3, fT4, TSH, IGF-1, FSH, LH, estradiol, DHEAS, testosterone, cortisol), bone metabolism (calcium, phosphorus, 25-OH Vitamin D, PTH, $\beta$-crosslaps, bone alkaline phosphatase) and total antioxidant capacity (TAC), expressed as LAG (latency time in radical species appearance using spectrophotometric method). BMD was assessed by DEXA. Group A patients exhibited significantly lower levels of IGF-1 (159.76 \pm 14.79 vs. $220.05 \pm 15.25 \mathrm{ng} / \mathrm{ml})$ and osteocalcin (17.51 \pm 1.14 vs. $21.49 \pm 1.56 \mathrm{ng} / \mathrm{ml})$; LAG values were significantly higher in $\mathrm{A}(66.33 \pm 1.74 \mathrm{~s}) \mathrm{vs}$. $\mathrm{B}(54.62 \pm 1.74 \mathrm{~s})$. A significant direct correlation was found between both IGF-1 and fT 3 with osteocalcin $\left(r^{2}=0.22, p=0.0049\right.$ and $r^{2}=0.34$, $\mathrm{p}=0.0001$, respectively). No difference in LAG between groups according to IGF-1 were found. These data show a correlation between altered bone turnover and low fT3, which is highly prevalent in FHA. Low fT3 levels may contribute to reduced BMD. Oxidative stress could be the link underlying different bone turnover pattern and endocrine dysfunction in FHA.

\section{Introduction}

Secondary amenorrhea, defined as a 3 months absence of menstruation in a previously cycling woman, occurs in 3-5\% of women, and $20-35 \%$ of them are affected by functional hypothalamic amenorrhea (FHA) [1]. FHA is a form of chronic anovulation, which is not related to an identifiable organic cause [2], classified as a hy- pogonadotropic hypogonadism [3]. It is a "functional" condition because the correction of causal behavioral factors, such as stress, anxiety, excessive physical exercise and weight loss, can normalize ovulatory function.

It is known that the main feature is a reduction in GnRH (gonadotropin-releasing hormone) signal, which manifests as reduced 
lutenizing hormone (LH) pulse frequency [3] and follicle-stimulating hormone (FSH) levels insufficient to maintain an adequate folliculogenesis and ovulatory function as demonstrated by $\mathrm{GnRH}$ or gonadotropin exogenous use, which can guarantee folliculogenesis [3]. The consequent hypoestrogenism has a negative mark on different aspects of female health $[4,5]$.

However, other endocrine and metabolic dysfunction, such as an activation in hypothalamic-pituitary-adrenal axis (HPA) $[4,6]$, assayable serum growth hormone $(\mathrm{GH})$ levels during night-time and lower $24 \mathrm{~h}$ prolactin (PRL) levels [7], low serum insulin and insulin-like growth factor 1 (IGF-1), have been described. Moreover, a consequence of the activation of HPS axis is the enhanced production of endogenous opioid peptides (mostly endorphins, enkephalins, and dynorphin) [8] and other neurohormonal factors involved in stress response such as dopamine, neurotensin, serotonin [9] and several neuropeptides: substance $P$, neuropeptide $Y$ (NPY), and calcitonin gene-related peptide (CGRP) [4].

An alteration of hypothalamic-pituitary-thyroid axis (HPT) has also been detected including a low-to-normal level of thyrotropin, a low level of triiodothyronine, and increased level of reverse triiodothyronine [5], a condition defined "non-thyroidal illness syndrome" (NTIS). Usually, this condition, described in other chronic disease, is considered an adaptive mechanism and its treatment is still debated. However, it could contribute to the clinical presentation of FHA.

One of the most frequent event is the decrease of bone mass density, related to an increase of fracture risk; it is possible to affirm that osteopenia and osteoporosis are the main long-term complications of FHA [5]. This problem has a great social relevance when considering the cost of morbidity and mortality, which usually are considered only in ageing population [10]. The prevalence in young population, before peak bone density is reached, strengthens the interest of the topic.

It is well known that improper diet, leading to low calcium and vitamin D3 intake, malnutrition, excessive exercises [11] and especially estrogens play a critical role in bone metabolism [12]. The result of estrogens activity is the activation bone remodeling units, an enhancement in bone formation and a suppression of bone resorption [12]. Androgens, fT3, GH, and IGF-1 are the other hormones, which exert a positive influence on bone formation [13], even if their role is not so clear as estrogens one.

One of the possible mechanism through which these hormones can exert their activity on bone may probably be oxidative stress (OS). OS is caused by the unbalancing between production of free radicals, molecules characterized by high reactivity due to one or more unpaired electrons in the external orbital, and antioxidant defenses in the biological systems [14]. Radical oxygen species (ROS) greatly influence the generation and survival of osteoclasts, osteoblasts, and osteocytes and loss of estrogens and androgens decrease defense against OS in bone [15]. On the other hand, different hormones are able to modulate antioxidant systems, as previously reviewed, in particular thyroid hormones [16] and NTIS have been related to OS.

The aim of this observational cohort study is therefore to evaluate the impact of hormonal alterations and antioxidant systems on bone turnover in FHA, with a particular focus on NTIS. In order to evaluate the impact of low fT3 on bone turnover parameters and antioxidant levels, we have divided the patients according to fT3 levels in 2 groups to explore the differences between low- and normal-fT3 patients, hy- pothesizing oxidative stress as a possible mechanism contributing to reduced bone mineral density (BMD) in such patients.

\section{Patients and Methods}

Subjects involved in this study were admitted to the University Hospital "Policlinico Gemelli" Department of Internal Medicine and were enrolled after being given an explanation of purposes and nature of the study, conducted in accordance with the Declaration of Helsinki, as revised in 2013. The study protocol was approved by Review Board of the "Institute of Medical Pathology" of our Hospital and written informed consent was obtained from all patients.

We included 43 patients with diagnosis of hypothalamic amenorrhea lasting at least 3 months, confirmed by typical endocrine picture (see below) and absent response to medroxyprogesterone administration, according to the Endocrine Society Practice Guidelines [2]. They were aged 15-34 years, with a BMI range $17.3-23.4 \mathrm{~kg} / \mathrm{m}^{2}$.

Criteria of exclusion were: Anorexia nervosa according to DSM V criteria [17], diabetes mellitus, liver or kidney chronic failure, corticosteroid therapy, hyperparathyroidism, obesity, malabsorption or other gastro-enteric diseases, and neurological diseases. Women with secondary amenorrhea due to other causes, specifically hyperprolactinemia, Cushing's syndrome, congenital adrenal hyperplasia, polycystic ovarian syndrome or primary ovarian failure, were excluded.

Patients were divided in 2 groups according to fT3 levels: group A (low fT3, $\mathrm{n}=22, \mathrm{fT} 3$ values $<2.4 \mathrm{pg} / \mathrm{ml}$ according to laboratory range), group $B$ (normal fT3, $n=21$, fT3 values $\geq 2.4 \mathrm{pg} / \mathrm{ml}$ ).

An endocrine evaluation including fT3, fT4, thyroid-stimulating hormone (TSH), IGF-1, follicle-stimulating hormone (FSH), luteinizing hormone (LH), estradiol (E2), dehydroepiandrosterone-sulfate (DHEAS), testosterone ( $\mathrm{T}$ ), and cortisol levels was performed; bone metabolic parameters were also evaluated $(25 \mathrm{OH}$-vitamin $\mathrm{D}$, calcium, phosphorus, parathormone (PTH), osteocalcin (OC), $\beta$-crosslaps, and bone alkaline phosphatase. For the evaluation of antioxidant systems, blood samples were collected at 08:00 AM, after overnight fast, immediately centrifuged and stored at $-80{ }^{\circ} \mathrm{C}$ until assayed, to evaluate Total Antioxidant Capacity (TAC). Finally, bone mineral density was assessed by DEXA.

The following methods were used for hormone assay: ElectroChemiluminescent method (ECLIA) for PTH (n.r. 14-72 pg/ml), OC (n.r. $10-45 \mathrm{ng} / \mathrm{ml}$ ), $\beta$-crosslaps (n.r. $0.2-0.7 \mathrm{ng} / \mathrm{ml}$ ); ChemoLuminescent Immunoassay for TSH (n.r. $0.35-2.80 \mu \mathrm{Ul} / \mathrm{ml}$ ), fT3 (n.r. 2.4$4.2 \mathrm{ng} / \mathrm{ml}$ ), fT4 (n.r. 8.5-16.5 pg/ml), IGF-1 (n.r. 80-330 ng/ml), FSH (2.5-11 mU/ml), LH (2.5-10 mU/ml), E2 (normal values <44 ng/ml), DHEAS (n.r. 800-3500 ng/ml), T (n.r. 0.20-2.00 ng/ml), cortisol (n.r $60-220 \mathrm{ng} / \mathrm{ml}$ ), vitamin $D($ n.r. $31-100 \mathrm{ng} / \mathrm{ml})$, bone alkaline phosphatase (n.r 5.5-25 $\mathrm{g} / \mathrm{l})$, and Chemiluminescent Microparticle ImmunoAssay (CMIA) for LH (2.5-15 mU/ml). Calcium was measured with Arsenazo III method, phosphate with colorimetric assay.

As IGF-1 is concerned, we also calculated the median value, according to sex and age, using reference provided by Liason ${ }^{\circledR}$ Analizer producer (DiaSorin, Vercelli, Italy), to classify patient with low or normal IGF-1.

Total Antioxidant Capacity (TAC) was evaluated, with a modification of the method developed by Rice-Evans and Miller [18], as previously described [19]. The method is based on the antioxidants inhibition of the absorbance of the radical cation ABTS. ${ }^{+}$formed by 
interaction between ABTS [2,2'-azinobis(3-ethylbenzothiazoline-6-sulfonic acid) diammonium salt; $150 \mu \mathrm{M}$ ] and ferrylmyoglobin radical species, generated by activation of metamyoglobin $(2.5 \mu \mathrm{M})$ with $\mathrm{H}_{2} \mathrm{O}_{2}(75 \mu \mathrm{M})$.

Aliquots of the frozen plasma were thawed at room temperature and $10 \mu$ l of the samples was tested immediately. The manual procedure was used with only minor modifications, that is, temperature at $37{ }^{\circ} \mathrm{C}$ to be in more physiological conditions and each sample assayed alone to carefully control timing and temperature. The reaction was started directly in cuvette through $\mathrm{H}_{2} \mathrm{O}_{2}$ addition after 1 min equilibration of all other reagents (temperature control by a thermocouple probe, model $1408 \mathrm{~K}$ thermocouple (Digitron Instrumentation Ltd, Scunthorpe, UK) and followed for $10 \mathrm{~min}$ under continuous stirring, monitoring at $734 \mathrm{~nm}$, typical of the spectroscopically detectable ABTS. ${ }^{+}$. The presence of chain-breaking antioxidants induces a lag time (the Lag phase) in the accumulation of ABTS. + whose duration is proportional to the concentration of this type of antioxidants. Antioxidant capacity afforded by chain-breaking antioxidants is expressed as length of Lag phase (LAG, sec). Trolox, a water-soluble tocopherol analogue, was used as a reference standard and assayed in all experiments to control the system. Absorbance was measured with an Agilent 8453 UV/ Vis spectrophotometer (Palo Alto, CA, USA) equipped with a cuvette stirring apparatus and a constant temperature cell holder. Measurements of $\mathrm{pH}$ were made with a PHM84 Research pH meter (Radiometer, Copenhagen, Denmark); the electrode response was corrected for temperature. Unless stated differently, experiments were repeated 2-3 times; qualitatively similar results were obtained with individual values varying $<8 \%$. In the Lag mode, the assay mainly measures non-proteic and non-enzymatic antioxidants that are primarily extracellular chainbreaking antioxidants, such as ascorbate, urate, and glutathione.

BMD was assessed at the neck of the right hip femur and at the lumbar spine through DXA scan with Hologic ${ }^{\circledR}$ Discovery A (Hologic, Inc., Bedford, MA, USA). Only 4 patients were under 20 years of age and a Z-score <-2 was used to define "low bone density for chronologic age" [20]; in other patients T-score was used to define osteopenia/osteoporosis.

\section{Statistical analysis}

Mean and Standard Error (SEM) were used to describe quantitative variables. The Mann-Whitney U-Test was used to evaluate differences in hormonal and bone metabolism parameters between the 2 groups. Analysis of variance (ANOVA) was used for comparison among different groups. Spearman correlation coefficient was used to investigate the association between $\mathrm{fT} 3$ or IGF- 1 vs. OC. A value of $p<0.05$ was considered statistically significant and the analysis was performed using Stata 13.

\section{Results}

In our cohort, 22 patients exhibited low fT3 concentration (group A), while 21 patients showed a value in the normal range (group B). - Table 1 depicts population general features, showing no significant differences in age, BMI, time of onset and length of amenorrhea at the examination.

DEXA showed a worse condition in group A, as illustrated in > Fig. 1 (mean femur neck $\mathrm{T}$-score ranged from -0.40 to -2.20 in group $A$, from +1.80 to -1.60 in group $B$; mean lumbar T-score from -0.60 to -3.40 in group $A$, from +0.20 to -2.90 in group $B$ ), defining, in group $A$, $34 \%$ osteoporosis and $66 \%$ osteopenia, and, in group B, $8 \%$ osteoporosis, $75 \%$ osteopenia and $17 \%$ normal bone density. In such evaluation only 4 patients, who were aged $<20$, were not included.

- Table 2 shows mean \pm SEM levels of the studied hormones and bone metabolism parameters; in > Fig. 2, IGF-1 and OC values in 2 groups are also shown. Both parameters were significantly lower in group A. Moreover, a significant correlation was present when plotting fT3 and IGF- 1 values $\left(r^{2}=0.29 ; p=0.0003\right)$. The 2 groups also significantly differed in cortisol values ( $\triangleright$ Table 1 ). Both fT3 and IGF-1 significantly correlated with OC levels ( $\bullet$ Fig. $\mathbf{3}$ ).

Concerning oxidative parameters, group A presented higher levels of LAG than group B ( $\triangleright$ Fig. 4). On the contrary, when dividing patient according to IGF-1 levels (separating patients IGF-1 levels over or under the median for each age), no differences in LAG values were observed.

Finally, interesting results were obtained with a further stratification in 3 groups of patients according to fT3 levels, separating group B in 2 subgroups according to median value of $\mathrm{fT} 3$ observed in this one $(2.6 \mathrm{pg} / \mathrm{ml})$. In fact, among 21 patients, 12 exhibited low-normal values $(2.4-2.6 \mathrm{pg} / \mathrm{ml})$ and 9 normal values. Mean OC level in these 3 subgroups are reported in ( $>$ Fig. 1S) (supplementary material). We found that also in low-normal fT3 patients OC levels were significantly lower than in normal fT3 patients.

\section{Discussion}

Our data confirm multi-hormonal derangement in FHA, with negative impact on bone metabolism, according to literature [3-9], adding some new information on their reciprocal influence and regulation on antioxidant systems.

- Table 1 Mean \pm SEM of population general features.

\begin{tabular}{|l|l|l|l|l|}
\hline & Age (years) & BMI $\mathbf{( k g / \mathbf { m } ^ { 2 } )}$ & Time of onset (years) & Length of amenorrhea (months) \\
\hline Group A & $17-34(25.6 \pm 1.2)$ & $19.7 \pm 0.35$ & $24.14 \pm 1.6$ & $10.15 \pm 1.1$ \\
\hline Group B & $19-34(26.1 \pm 1)$ & $19.45 \pm 0.52$ & $24.78 \pm 1.4$ & $8.2 \pm 0.7$ \\
\hline P & NS & NS & NS & NS \\
\hline
\end{tabular}



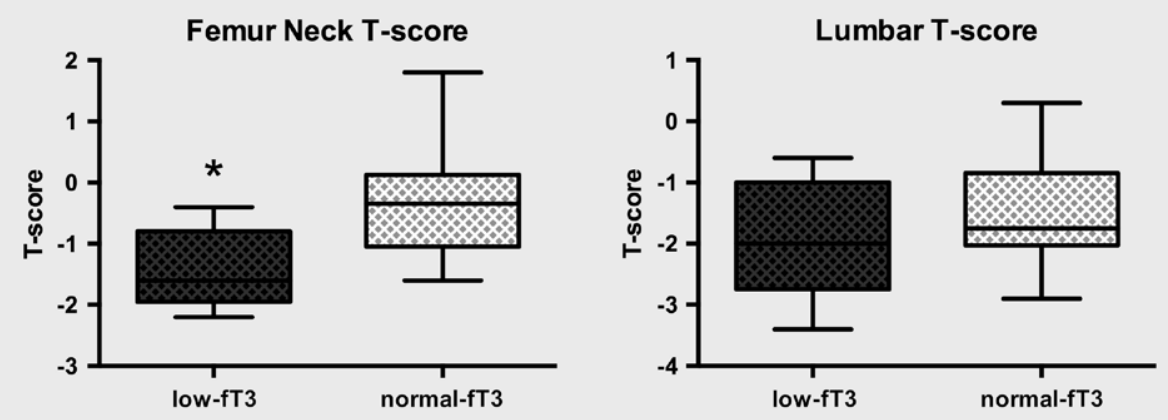

- Fig. 1 Box plot showing T-score values at lumbar and femoral level in our patients aged $>20$ years. ${ }^{*} p<0.05$.

- Table 2 Mean \pm SEM of hormonal parameters and bone metabolism parameters.

\begin{tabular}{|c|c|c|c|}
\hline & Group A & Group B & $\mathbf{p}$ \\
\hline fT3 $(p g / m l)$ & $2.19 \pm 0.04$ & $2.68 \pm 0.06$ & $<0.05$ \\
\hline $\mathrm{fT} 4$ (pg/ml) & $9.20 \pm 0.24$ & $10.18 \pm 0.27$ & NS \\
\hline $\mathrm{TSH}(\mu \mathrm{UI} / \mathrm{ml})$ & $1.41 \pm 0.14$ & $1.68 \pm 0.22$ & NS \\
\hline $\mathrm{FSH}(\mathrm{mUl} / \mathrm{ml})$ & $5.22 \pm 0.45$ & $5.70 \pm 0.39$ & NS \\
\hline $\mathrm{LH}(\mathrm{mUI} / \mathrm{ml})$ & $2.16 \pm 0.43$ & $3.64 \pm 0.64$ & NS \\
\hline E2 (pg/ml) & $25.05 \pm 2.57$ & $32 \pm 3.97$ & NS \\
\hline DHEAS (ng/ml) & $2513 \pm 230.79$ & $2416.53 \pm 245.55$ & NS \\
\hline $\mathrm{T}(\mathrm{ng} / \mathrm{ml})$ & $0.44 \pm 0.06$ & $0.42 \pm 0.13$ & NS \\
\hline Cortisol (ng/ml) & $163.86 \pm 13.52$ & $116.84 \pm 7.51$ & $<0.05$ \\
\hline Vitamin D (ng/ml) & $28.07 \pm 2.49$ & $28.84 \pm 1.60$ & NS \\
\hline Calcium (mg/dl) & $9.66 \pm 0.06$ & $9.71 \pm 0.06$ & NS \\
\hline Phosphorus (mg/dl) & $3.42 \pm 0.11$ & $3.61 \pm 0.09$ & NS \\
\hline PTH (pg/ml) & $35.65 \pm 2.42$ & $40.32 \pm 3.50$ & NS \\
\hline$\beta$-Cross-laps (ng/ml) & $0.52 \pm 0.04$ & $0.53 \pm 0.04$ & NS \\
\hline Bone alkaline phosphatase $(\mu \mathrm{g} / \mathrm{l})$ & $10.85 \pm 4.62$ & $23.18 \pm 7.37$ & NS \\
\hline
\end{tabular}

In our cohort, a significant number of patients showed low fT3 levels. Data of BMD in our low-fT3 patients showed a worse picture in comparison to normal ones. Low fT3 syndrome, usually considered as an adaptive mechanism, therefore not to be treated by replacement therapy, as in other condition of systemic diseases [21], could be responsible for negative consequences in the bone, representing a negative worsening factor in synergy with low IGF-1 and high cortisol levels.

When dividing patients according to fT3 levels, we found significantly lower levels of OC, IGF-1, and significantly increased cortisol levels. On the contrary, estradiol levels did not differ between the 2 groups. Moreover, a significant correlation was present between fT3 and IGF-1. Both IGF-1 and fT3 significantly correlated with osteocalcin, accordingly to a positive action of both on osteoblastic activity.

A condition of $\mathrm{GH}$ resistance is present in patients affected by anorexia nervosa [22]. Even our patients showed low levels of IGF-1, significantly correlated with fT3 and osteocalcin. Although GH itself was not measured in our study, the mechanism involved in FHA is probably the same. Low IGF-1 levels are associated with an increased fracture risk both in men [23] and women [24]. Both $\mathrm{GH}$, via direct action, and locally produced IGF-1 exert independent, but integrated effects on skeletal cytotypes; the cellular machinery is even more elaborate when considering the modulatory activity of IGF-binding proteins [25]. Moreover estrogens have profound interactions with such systems, also explaining sex-related differences in bone metabolism [26]. Recently, other mechanisms have been claimed to explain $\mathrm{GH}$ resistance in anorexia nervosa [27], such as increased FGF-21, low insulin and increased ghrelin, the increased expression of the deacetylase Sirtuin-1; all these factor underline the link between metabolic request and defense mechanisms. If such mechanisms operate also in other forms of FHA is not known. Anyhow, in our study IGF-1 levels correlated with 

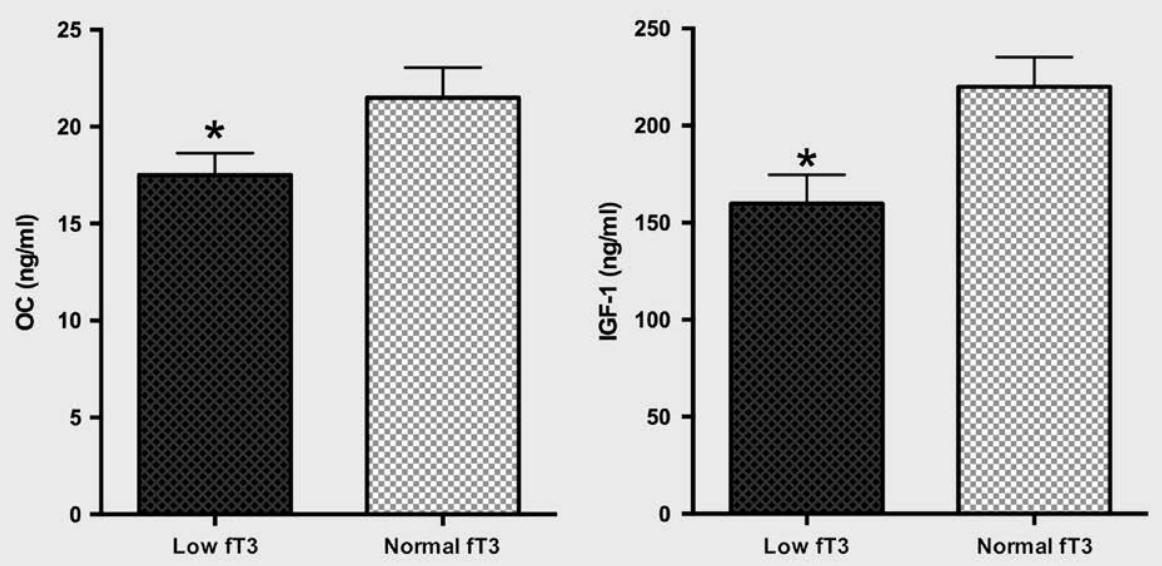

- Fig. 2 Mean \pm SEM values of OC (left panel) and IGF-1 (right panel) in the 2 groups. ${ }^{*} \mathrm{p}<0.05$.
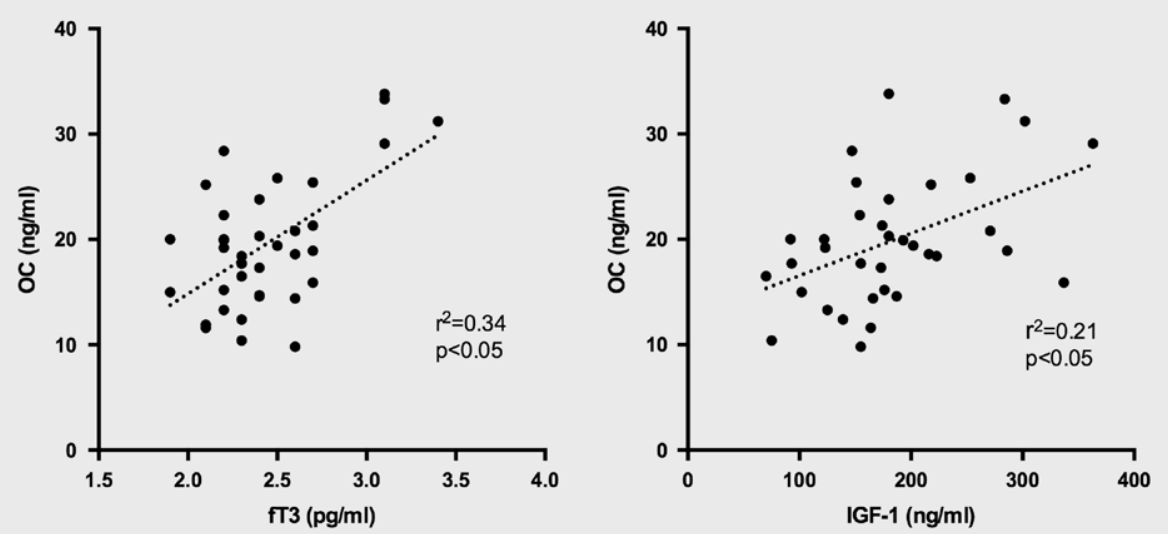

- Fig. 3 Correlations between OC and fT3 (left panel) and IGF-1 (right panel).
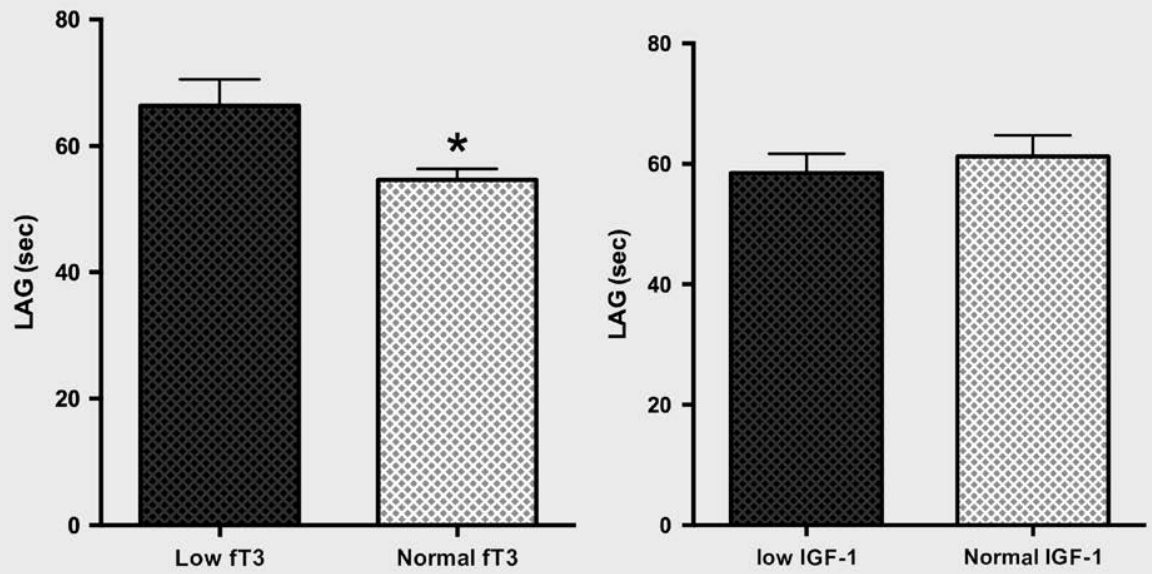

- Fig. 4 Mean \pm SEM values of LAG in the 2 groups in accordance to $\mathrm{fT} 3$ values (left panel) and IGF- 1 (right panel). ${ }^{*} \mathrm{p}<0.05$, LAG: Duration of latency phase before the appearance of radical species (see text for explanation). 
osteocalcin, suggesting a promoting action on osteoblast production. IGF-1 and OC show parallel patterns in different models in literature $[28,29]$, however, studies about skeletal maturation suggest a triggering action on OC synthesis [30].

The effects of low fT3 are less clear. Osteoblasts have receptors for thyroid hormone [31]; experimental animals KO for thyroid receptors have a reduced trabecular BMD and high marrow fat [32], resembling alterations of hypoestrogenic women. Studies on fT3 actions on osteoblasts are contradictory [33], especially when considering that both hypo- and hyperthyroidism can induce osteoporosis; but they globally suggest positive fT3 effect on differentiation and activity of osteoblasts, while the actions on osteoclasts seem to be indirect. As differentiation and proliferation is concerned, induction of IGF-1, IGFBP-2, and -4, FGF receptors and signaling are stimulated by fT3 [34-36]. About osteoblast activity, fT3 has been demonstrated to stimulate type I collagen synthesis and post-translational modification, alkaline phosphatase expression, osteopontin and osteocalcin synthesis and secretion [37,38]. These experimental data well fit with the direct correlation between fT3 and osteocalcin in our patients. The effect on osteoclasts, mediated by osteoprotegerin, are still controversial [33]. In the model of FHA, the problem could be related to local deiodination rather than low circulating fT3 levels; in such sense, studies performed in mice, with deletion of type 2 deiodinases gene (dio2) suggest the key role of fT3 in osteoblast activity [39].

Cortisol levels, increased in group A could also have a relevant role in our findings, directly contributing to reduced bone mineral density, but also influencing the conversion of I-thyroxine to fT3 [40]. Both increased cortisol and low fT3 could express a worse hypothalamic derangement and metabolic condition of this group. Whatever the mechanism, cortisol levels were not themselves correlated significantly neither to OC, nor to $\mathrm{fT} 3$ and LAG.

A great importance in negative skeletal condition is usually attributed to the state of hypoestrogenism [22], since estrogens exert a triple action, activating bone remodeling units, suppressing bone reabsorption and stimulating bone formation [12]. Osteoclastic activity is inhibited by different mechanisms [41], including inhibition of RANKL production and increased osteoprotegerin gene expression [42]. Other cytokines, favoring bone reabsorption, such as macrophage-colony stimulating factor (M-CSF), interleukins 1 and 6, tumor necrosis factor $\alpha$ (TNF- $\alpha$ ), are inhibited by estrogens. They indirectly help osteoblastic activity, decreasing sclerostin (which inhibits osteoblastic WNT signaling) [43] and preadipocyte factor-1 (Pref-1), member of EGF family, which inhibits the differentiation of osteoblasts from mesenchimal progenitor cells [44]. Finally, estrogens stimulate other effectors, such as bone morphogenetic protein 6-BMP6 and transforming growth factor $\beta$; but also IGF-1, locally produced in the bone after GH stimulation, is augmented by estrogens. They also increase the expression of vitamin D receptors [45]. Such complex and pleomorphic action can obviously have an impact on skeleton and some studies suggest a minimal threshold of $40-50 \mathrm{pg} / \mathrm{ml}$ to observe effects on bone [46]. However, we did not find differences in estradiol in our 2 groups, emphasizing additional influences of other systems.

Even if low fT3 and IGF-1 could have a synergic effect on bone, our data suggest that they could work with different mechanisms, in fact only fT3 seemed to influence antioxidant systems in our pop- ulation. Greater LAG values in low-fT3 patients suggest a greater oxidative stress in such group with a compensatory increase in anti-oxidant systems. Previously we have shown that thyroid hormones profoundly affect the antioxidant defense of the body, leading to a condition of oxidative stress [16]. Both hyper- and hypothyroidism can induce oxidative stress; but in the case of hypothyroidism, the low fT3 condition could worsen the oxidative status of the cell, with a vicious circle. Mechanism of competition on glutathione, which is a cofactor of deiodinases, but also strong antioxidant, have been claimed. Also growth hormone deficiency/ resistance is associated with oxidative stress, even if with a different pattern of antioxidants [47]. While in this study we did not find differences in TAC in relation to IGF-1, the values of LAG were significantly different in groups with low or normal fT3. The augmented LAG could express a compensatory mechanism to a greater oxidative stress, directly influencing bone metabolism, as shown in other in vivo models [48].

Under this profile, it could be of interest that also patients with low-normal fT3 have low osteocalcin level, suggesting that the biochemical mechanisms operating at cellular levels can be very precocious, requiring therefore a special attention.

In conclusion, osteopenia/osteoporosis in FHA should be considered a multifactorial problem. While no doubts exist that low estrogens and vitamin D deficiency can play a pivotal role, other hormonal derangement, such as low fT3 and IGF-1, although by different mechanisms, could be considered in such condition.

Nevertheless, there are two main potential restrictions to consider in the present study. First, the number of subjects in both groups is slightly small, so its statistical power is limited, thus our findings will need to be confirmed in a larger population. Second, this cohort-study and the power analysis cannot draw a cause-effect conclusion about oxidative stress and osteoporosis in patients affected by FHA.

\section{Conflict of Interest}

The authors declare that they have no conflict of interest.

\section{References}

[1] The Practice Committee of the American Society for Reproductive Medicine. Current evaluation of amenorrhea. 2008 Compend Pract Comm Reports 2008; 90: S219-S225

[2] Gordon CM, Ackerman KE, Berga SL et al. Functional hypothalamic amenorrhea: An endocrine society clinical practice guideline. J Clin Endocrinol Metab 2017; 102: 1413-1439

[3] Berga SL, Mortola JF, Girton L et al. Neuroendocrine aberrations in women with functional hypothalamic amenorrhea. J Clin Endocrinol Metab 1989; 68: 301-308

[4] Meczekalski B, Podfigurna-Stopa A, Warenik-Szymankiewicz A et al. Functional hypothalamic amenorrhea: Current view on neuroendocrine aberrations. Gynecol Endocrinol 2008; 24: 4-11

[5] Meczekalski B, Katulski K, Czyzyk A et al. Functional hypothalamic amenorrhea and its influence on women's health. J Endocrinol Invest 2014; 37: 1049-1056 
[6] Berga SL, Daniels TL, Giles DE. Women with functional hypothalamic amenorrhea but not other forms of anovulation display amplified cortisol concentrations. Fertil Steril 1997; 67: 1024-1030

[7] Laughlin GA, Dominguez CE, Yen SSC. Nutritional and endocrine-metabolic aberrations in women with functional hypothalamic amenorrhea. J Clin Endocrinol Metab 1998; 83: 25-32

[8] Kavushansky A, Kritman M, Maroun M et al. $\beta$-Endorphin degradation and the individual reactivity to traumatic stress. Eur Neuropsychopharmacol 2013; 23: 1779-1788

[9] Bérubé P, Laforest S, Bhatnagar $S$ et al. Enkephalin and dynorphin mRNA expression are associated with resilience or vulnerability to chronic social defeat stress. Physiol Behav 2013; 122: 237-245

[10] Kling JM, Clarke BL, Sandhu NP. Osteoporosis prevention, screening, and treatment: A review. J Womens Health (Larchmt) 2014; 23: 563-572

[11] De Souza MJ, Williams NI. Physiological aspects and clinical sequelae of energy deficiency and hypoestrogenism in exercising women. Hum Reprod Update 2004; 10: 433-448

[12] Khosla S. Update on estrogens and the skeleton. J Clin Endocrinol Metab 2010; 95: 3569-3577

[13] Seeman E. Pathogenesis of bone fragility in women and men. Lancet 2002; 359: 1841-1850

[14] Halliwell B, Gutteridge JMC. Free Radicals in Biology and Mededicine. In Halliwell B, Gutteridge JMC. (Eds.). Free radicals in biology and medicine. 3rd Edition Oxford University Press; Oxford: 1999: 1-25

[15] Manolagas SC. From estrogen-centric to aging and oxidative stress: A revised perspective of the pathogenesis of osteoporosis. Endocr Rev 2010; 31: 266-300

[16] Mancini A, Festa R, Di Donna V et al. Hormones and antioxidant systems: Role of pituitary and pituitary-dependent axes. J Endocrinol Invest 2010; 33: 422-433

[17] American Psychiatric Association. Diagnostic and Statistical Manual of Mental Disorders DSM-V. Washington D. C.: 2013

[18] Rice-Evans C, Miller NJ. Total antioxidant status in plasma and body fluids. Methods Enzymol 1994; 234: 279-293

[19] Mancini A, Leone E, Festa R et al. Evaluation of antioxidant systems (coenzyme Q10 and total antioxidant capacity) in morbid obesity before and after biliopancreatic diversion. Metabolism 2008; 57: 1384-1389

[20] Baroncelli Gl, Bertelloni S, Sodini F et al. Osteoporosis in children and adolescents etiology and management. Pediatr Drugs 2005; 7: 295-323

[21] Mancini A, Di Segni C, Raimondo S et al. Thyroid hormones, oxidative stress, and inflammation. Mediators Inflamm 2016; Article ID 6757154 http://dx.doi.org/10.1155/2016/6757154

[22] Misra M, Klibanski A. Anorexia nervosa and bone. J Endocrinol 2014; 221: R163-R176

[23] Ohlsson C, Mellström D, Carlzon D et al. Older men with low serum IGF-1 have an increased risk of incident fractures: The MrOS Sweden study. J Bone Miner Res 2011; 26: 865-872

[24] Sugimoto T, Nishiyama K, Kuribayashi F. Serum levels of insulin-like growth factor (IGF) I, osteoporotic patients with and without spinal fractures. J Bone Min Res 1997; 12: 1272-1279

[25] Locatelli V, Bianchi VE. Effect of GH/IGF-1 on bone metabolism and osteoporsosis. Int J Endocrinol 2014; 2014235060 doi:10.1155/2014/235060

[26] Vandenput L, Boonen S, Van Herck E et al. Evidence from the aged orchidectomized male rat model that $17 \beta$-estradiol is a more effective bone-sparing and anabolic agent than $5 \alpha$-dihydrotestosterone. J Bone Miner Res 2002; 17: 2080-2086

[27] Fazeli PK, Klibanski A. Anorexia nervosa and bone metabolism. Bone 2014; 66: 39-45
[28] Wang Y, Bikle DD, Chang W. Autocrine and paracrine actions of IGF-I signaling in skeletal development. Bone Res 2013; 1: 249-259

[29] Ghodsi M, larijani B, Keshtkar AA et al. Mechanisms involved in altered bone metabolism in diabetes: A narrative review. J Diabetes Metab Disord 2016; 15: 52

[30] Tripathi T, Gupta P, Rai P et al. Osteocalcin and serum insulin-like growth factor- 1 as biochemical skeletal maturity indicators. Prog Orthod 2017; 18: 30

[31] Abu EO, Bord S, Horner A et al. The expression of thyroid hormone receptors in human bone. Bone 1997; 21: 137-142

[32] Kindblom JM, Gevers EF, Skrtic SM et al. Increased adipogenesis in bone marrow but decreased bone mineral density in mice devoid of thyroid hormone receptors. Bone 2005; 36: 607-616

[33] Williams GR. Thyroid hormone actions in cartilage and bone. Eur Thyroid J 2012; 3-13

[34] Xing W, Govoni KE, Donahue LR et al. Genetic evidence that thyroid hormone is indispensable for prepubertal insulin-like growth factor-I expression and bone acquisition in mice. J Bone Miner Res 2012; 27: 1067-1079

[35] Milne M, Quail JM, Rosen C] et al. Insulin-like growth factor binding proteins in femoral and vertebral bone marrow stromal cells: expression and regulation by thyroid hormone and dexamethasone. J Cell Biochem 2001; 81: 229-240

[36] Stevens DA, Harvey CB, Scott A] et al. Thyroid hormone activates fibroblast growth factor receptor-1 in bone. Mol Endocrinol 2003; 17 : 1751-1766

[37] Varga F, Rumpler M, Zoehrer R et al. T3 affects expression of collagen I and collagen cross-linking in bone cell cultures. Biochem Biophys Res Commun 2010; 402: 180-185

[38] Gouveia CH, Schultz J], Bianco AC et al. Thyroid hormone stimulation of osteocalcin gene expression in ROS 17/2.8 cells is mediated by transcriptional and post-transcriptional mechanisms. J Endocrinol 2001; 170: 667-675

[39] Bassett JHD, Boyde A, Howell PGT et al. Optimal bone strength and mineralization requires the type 2 iodothyronine deiodinase in osteoblasts. Proc Natl Acad Sci USA 2010; 107: 7604-7609

[40] Mastorakos G, Pavlatou M. Exercise as a stress model and the interplay between the hypothalamus-pituitary-adrenal and the hypothalamus-pituitary-thyroid axes. Horm Metab Res 2005; 37: 577-584

[41] Riggs BL. The mechanisms of estrogen regulation of bone resorption. J Clin Invest 2000; 106: 1203-1204

[42] Mircea CN, Lujan ME, Pierson RA. Metabolic fuel and clinical implications for female reproduction. J Obstet Gynaecol Canada 2007; 29: 887-902

[43] Mödder Ull, Clowes JA, Hoey K et al. Regulation of circulating sclerostin levels by sex steroids in women and in men. J Bone Miner Res 2011; 26: 27-34

[44] Fazeli PK, Bredella MA, Freedman L et al. Marrow fat and preadipocyte factor- 1 levels decrease with recovery in women with anorexia nervosa. J Bone Miner Res 2012; 27: 1864-1871

[45] Taes Y, Lapauw B, Vandewalle $S$ et al. Estrogen-specific action on bone geometry and volumetric bone density: Longitudinal observations in an adult with complete androgen insensitivity. Bone 2009; 45: 392-397

[46] Crandall C], Tseng C-H, Karlamangla AS et al. Serum sex steroid levels and longitudinal changes in bone density in relation to the final menstrual period. J Clin Endocrinol Metab 2013; 98: E654-E663

[47] Mancini A, Di Segni C, Bruno C et al. Oxidative stress in adult growth hormone deficiency: Different plasma antioxidant patterns in comparison with metabolic syndrome. Endocrine 2018; 59: 130-136

[48] Mancini A, Raimondo S, Di Segni C et al. Comparison of plasma antioxidant levels in middle-aged and old male with idiopatic osteoporosis: Preliminary data. Eur Rev Med Pharmacol Sci 2014; 18: 2013-2019 